santalene, $\mathrm{C}_{15} \mathrm{H}_{24}$ über (terpentinartig riechende Flüssigkeiten). Das $\alpha$-Isosantalen siedet bei $255-56^{\circ} \mathrm{C}$. und zeigt $[\alpha]_{D}+0,2^{\circ}$, das $\beta$-Isosantalen bei $259-60^{\circ}$ C., $[\alpha]^{\mathrm{D}}+6,1^{\circ}$. Die beiden Santalole sind primäre Alkohole der Formel $\mathrm{C}_{15} \mathrm{H}_{26} \mathrm{O}$. Die Konstanten ihrer Esterifizierungsgeschwindigkeiten und -grenzen sind für die $\alpha-V e r-$ bindung 41,7 und 68,5 , für die $\beta$-Verbindung 43,6 und 69 .

Verf. bemerkt zum Schluss, dass infolge eines Irrtums das spez. Gewicht des Sandelholzöles in seiner ersten Arbeit (1. c.) fälschlich mit 0,9684 anstatt 0,9871 bei $0^{\circ} \mathrm{C}$. angegeben worden sei.

Wie man sieht, kommt Guerbet bei ungefähr gleicher Arbeitsweise bezüglich des $\alpha$-Santalols in zwei Punkten zu wesentlich anderen Resultaten wie ich. Nach ihm ist das $\alpha$-Santalol ein schwach links drehender Sesquiterpenalkohol $\mathrm{C}_{15} \mathrm{H}_{26} \mathrm{O}$; nach meiner Untersuchung dreht derselbe schwach rechts und hat die Formel $\mathrm{C}_{15} \mathrm{H}_{24} \mathrm{O}$. Ich glaube, dass Herr Guerbet doch noch nicht lange genug fraktioniert hat, um den Gehalt des stark links drehenden $\beta$-Santalols im $\alpha$-Santalol auf ein solches Minimum zu reduzieren, dass die Rechtsdrehung des letzteren zum Vorschein kam. Da ferner weder die Richtigkeit der Formel $\mathrm{C}_{15} \mathrm{H}_{26} \mathrm{O}$ für den $\alpha$-Alkohol, noch die der Formel $\mathrm{C}_{15} \mathrm{H}_{24}$ für das aus ersterem abgespaltene $\alpha$-Isosantalen durch Analysen bestätigt wird, so halte ich meine oben begrtindete Ansicht tiber die Zusammen. setzung des $\alpha$-Santalols aufrecht.

\title{
II. Zur Kenntnis des ostindischen Sandelholzöles. ${ }^{1}$ )
}

Von Friedrich Müller.

(Eingegangen den 11. VI. 1900.)

Als Material für die nachstehend beschriebenen, im Laboratorium der Firma Heine \& $\mathrm{Co}_{0}$, Leipzig, ausgeführten Untersuchungen diente ein von der genannten Firma durch Dampfdestillation aus dem zerkleinerten Holze von Santalum album $L$. gewonnenes Oel (ostind. Sandelholzöl). Indessen ging ich nicht direkt von dem so erhaltenen rohen Oele aus, sondern von zwei besonders interessierenden Bestandteilen desselben, einmal den sich bei der Destillation ergebenden Vor-

1) Die Publikation meiner bereits im Herbst 1899 durch äussere Umstände unterbrochenen Versuche erfolgt in diesem noch nicht völlig abgerundeten Zustande infolge verschiedener Veröffentlichungen von anderer Seite über denselben Gegenstand. 
läufen des zu ca. $90 \%$ aus schweren flüchtiger Sesquiterpenalkoholen bestehenden Oeles und sodann von den im rohen Sandelholzöl teils in freiem Zustande, teils in Form von Estern vorkommenden sauren Komponenten desselben. Letztere wurden z. T. aus den Verseifungslaugen des Oeles gewonnen, die sich bei dem der genannten Firma patentierten Verfahren ${ }^{1}$ ) ergeben.

\section{Untersuchung der Vorläufe.}

Ich ging bei dieser Untersuchung von einem $3550 \mathrm{~g}$ wiegenden ersten Vorlauf aus, der bei der Wasserdampf-Destillation von etwa 300 Kilo Sandelholzöl erhalten war. Da dieser Vorlauf nicht ganz unerhebliche Mengen einer festen Säure in freiem Zustande, der Teresantalsäure enthielt, von der im 2. Teil dieser Abhandlung die Rede sein soll, so wurde er vor weiterer Verarbeitung zunächst mit ca. $3 \%$ iger ${ }^{2}$ ) Natronlauge durchgeschüttelt. Das so erhaltene, gut gewaschene, säurefreie Produkt wurde einer oft wiederholten, sorgfältigen Fraktionierung im Vakuum unterworfen. Nach successiver Fortlassung der etwa bis $135^{\circ}$ herab (15 mm Druck) siedenden Anteile, die nach einer privaten Mitteilung des Herrn H. v. Soden Körper von sesquiterpenartigem Charakter enthalten sollten ${ }^{B}$, die mich hier weniger interessierten, erhielt ich folgende Fraktionen:

\begin{tabular}{c|c|c|c|c}
\hline \hline Fraktion & Sdp. 15 mm & $\begin{array}{c}\text { Gewicht, } \\
\mathrm{g}\end{array}$ & $\begin{array}{c}\text { Spez. Gew. } \\
(150)\end{array}$ & $\begin{array}{c}\text { Opt. Drehung } \\
(100 \mathrm{~mm}- \\
\text { Rohr })\end{array}$ \\
\hline \hline 1 & $35-370$ & 100 & 0,872 & $-0030^{\circ}$ \\
2 & $37-40^{\circ}$ & 4 & 0,873 & - \\
3 & $40-45^{\circ}$ & 3 & 0,876 & - \\
4 & $45-50^{\circ}$ & 4 & 0,879 & - \\
5 & $50-60^{\circ}$ & 3 & 0,881 & $-10^{\circ}$ \\
6 & $60-70^{\circ}$ & 9 & 0,893 & $-20^{\circ}$ \\
7 & $70-80^{\circ}$ & 16 & 0,945 & $-20^{\circ} 15^{\circ}$ \\
8 & $80-90^{\circ}$ & 77 & 0,980 & \\
\hline
\end{tabular}

1) Behandlung des rohen Oeles mit heissem alkoholischen Alkali, D. R.-P. No. 110485.

2) Kohlensaure Alkalien sind weniger zu empfehlen, da sie die schwache Säure nicht quantitativ entfernen; in konzentrierten Laugen sind die Salze der Säure schwer löslich.

3) Die Elementar-Analyse einer derartigen Fraktion vom Siedepunkt ca. $262^{\circ}$ (gew. Druck) ergab auf $\mathrm{C}_{15} \mathrm{H}_{24}$ stimmende Zahlen. 


\begin{tabular}{|c|c|c|c|c|}
\hline Fraktion & Sidp. $15 \mathrm{~mm}$ & $\begin{array}{c}\text { Gewicht, } \\
\mathrm{g}\end{array}$ & $\begin{array}{c}\text { Spez. Gew. } \\
\left(15^{0}\right)\end{array}$ & $\begin{array}{l}\text { Opt. Drehung } \\
\text { (100 mm- } \\
\text { Rohr })\end{array}$ \\
\hline 9 & $90-100^{0}$ & 71 & 0,978 & $-35^{0} 45^{\prime}$ \\
\hline 10 & $100-110^{0}$ & 40 & 0,963 & $-29030^{\prime}$ \\
\hline 11 & $110-1200$ & 72 & 0,927 & $-13^{\circ} 35^{\prime}$ \\
\hline 12 & $120-122^{0}$ & 68 & 0,909 & $-11050^{\prime}$ \\
\hline 13 & $122-124^{0}$ & 214 & 0,902 & $-13^{0} 15^{\prime}$ \\
\hline 14 & $124-126^{0}$ & 334 & 0,898 & -19037 \\
\hline 15 & $126^{\circ}$ & 608 & 0,898 & $-17020^{\circ}$ \\
\hline 16 & $126-1280$ & 140 & 0,896 & $-24025^{\prime}$ \\
\hline 17 & $128-130^{0}$ & 27 & 0,896 & -300 \\
\hline 18 & $130-1320$ & 81 & 0,899 & $-29022^{\prime}$ \\
\hline 19 & $132-134^{0}$ & 62 & 0,899 & $-26010^{0}$ \\
\hline 20 & $134-136^{0}$ & 46 & 0,899 & $-26^{0}$ \\
\hline 21 & $136-138^{0}$ & 13 & 0,902 & $-29^{\circ} 25^{\prime}$ \\
\hline
\end{tabular}

Die niedrigeren Fraktionen zeigten einen äusserst intensiven pinen- und kampherartigen Geruch. Die Intensität desselben nimmt mit steigendem Siedepunkt stark ab und die über $120^{\circ}$ etwa siedenden Fraktionen besitzen nur noch einen sehr schwachen Geruch.

Wie aus den in der Tabelle zusammengestellten Eigenschaften der Fraktionen hervorgeht, sind darin anscheinend mindestens 3 verschiedene Körper enthalten, einer von sehr niedrigem Siedepunkt und niedrigem spez. Gewicht (Fraktion 1), einer von hohem spez. Gewicht und hoher opt. Drehung (Fraktionen 8 und 9) und ein bei ca. $126^{\circ}$ und $15 \mathrm{~mm}$ Druck siedender, mit dem spez. Gewicht von ca. 0,899, der die Hauptmenge der Fraktionen 13-21 ausmacht. Auch die letzteren Fraktionen bestehen nach ihren Eigenschaften zweifellos fast ausschliesslich aus den Sesquiterpenen, deren Anwesenheit im Sandelholzöl schon früher von $H$. v. Soden und mir konstatiert ist ${ }^{1}$ ), und die Guerbet ${ }^{2}$ ) jüngst in 2 Isomere, $\alpha$ - und $\beta$ - Santalen, zerlegt hat. Bei den Sesquiterpenen habe ich mich darauf beschränkt, zu konstatieren, dass die Hauptfraktion rom Siedepunkt $126^{\circ}$ (15 mm Druck) weder mit Nitrosylchlorid ${ }^{3}$ ) noch mit den Halogenen nach den gebräuchlichen Methoden feste Derivate lieferte.

1) H. v. Soden und F. Müller, Pharm. Ztg. 44, 258. - Vgl. auch die vorstehende Arbeit von $H$. v. Soden.

2) Guerbet, Comptes rendus, 1900, I, 417.

a) Guerbet (Comptes rendus 1900, I, 1324) hat inzwischen nach anderer Methode krystallisierte Santalennitrosochloride erhalten. 
Santen: $\mathrm{C}_{8} \mathrm{H}_{14}$.

Die beim Fraktionieren der Sandelholzölvorläufe erhaltene, am niedrigsten siedende Fraktion (Sdp. $35-37^{\circ}$ bei $15 \mathrm{~mm}$ Druck) wurde bei gewöhnlichem Druck destilliert und lieferte folgende Fraktionen:

\begin{tabular}{|c|c|c|c|c|c|c|}
\hline Sdp & 760 & $\mathrm{~mm}:$ & 1) & $139-140^{\circ}$ & $73 \mathrm{~g}$ & $\mathrm{I}_{15}=0,8715$ \\
\hline$\eta$ & 760 & $n$ & 2) & $140-142^{0}$ & 8 & $n=0,876$ \\
\hline$"$ & 760 & $"$ & 3) & $142-145^{0}$ & $4 n$ & $n=0,877$ \\
\hline " & 760 & $\eta$ & 4) & $145-150^{0}$ & 2 & $n=0,880$. \\
\hline
\end{tabular}

Ein Teil der so erhaltenen Hauptfraktion wurde nochmals, über metallischem Natrium, destilliert. Die Gesamtmenge siedete konstant bei $139-140^{\circ}$ und zeigte ein spez. Gewicht von 0,8710 bei $15^{\circ}$. Analysen (des über Natrium destillierten Produktes):

1) Subst. 0,1108: $\mathrm{H}_{2} \mathrm{O}=0,1166, \mathrm{CO}_{2}=0,3574 \mathrm{~g}$

2) $\quad 0,0980: \mathrm{H}_{2} \mathrm{O}=0,1024, \mathrm{CO}_{2}=0,3172$ n Gefunden: 1) $\mathrm{C}=87,97 \%, \mathrm{H}=11,69 \%$

2) $\mathrm{C}=88,27, \mathrm{H}=11,61$,

Berechnet für $\mathrm{C}_{8} \mathrm{H}_{14}: \mathrm{C}=88,52, \quad \mathrm{H}=11,48$, $" \quad " \mathrm{C}_{10} \mathrm{H}_{10}: \mathrm{C}=88,23, \quad \mathrm{H}=11,76$,

Nicht nur der Siedepunkt, sondern auch die Analysen sprechen fürr die Formel $\mathrm{C}_{9} \mathrm{H}_{14}$, welche durch die Analysen der folgenden Derivate sichergestellt wird. Es liegt somit ein neuer Kohlenwasserstoff vor, der ein nächst niedrigeres Homologes der Terpene $\mathrm{C}_{10} \mathrm{H}_{18}$ darstellt. In der That weist dieser Kohlenwasserstoff, den ich als $S$ anten bezeichnen will, in mehreren Punkten eine bemerkenswerte Aehnlichkeit namentlich mit dem Pinen auf. Sein Geruch nicht nur erinnert an Pinen mit einem Anklang an Kampher, sondern er liefert auch ein festes Salzsäureadditionsprodukt, das in Geruch und Verhalten frappante Aehnlichkeit mit Pinenchlorhydrat besitzt. Mit Nitrosylchlorid und salpetriger Säure giebt er feste Additionsprodukte.

Santen-Nitrosochloride.

Es gelingt leicht, aus dem Santen nach folgender Vorschrift ein prachtvoll kornblumenblaues Nitrosochlorid zu erhalten. In ein durch Kältemischung gut abgekühltes Gemenge von $5 \mathrm{~g}$ Santen, der gleichen Menge Eisessig und $6 \mathrm{~g}$ Aethylnitrit tropft man langsam $7,5 \mathrm{ccm}$ konzentrierte Salzsäure $(33 \%)$ worauf sich die Hauptmenge des Nitrosochlorids in schönen Krystallen abscheidet. Den in den Laugen gelösten Rest bekommt man, wenn man den Alkohol noch aus der Kältemischung schnell abdunstet. Man erhält so leicht aus dem Santen das gleiche Gewicht Nitrosochlorid, das man am besten schuell absaugt und mit wenig auf $-20^{\circ}$ abgekühltem Alkohol nachwäscht. Das blaue Nitrosochlorid zeichnet sich durch eine grosse Löslichkeit 
in fast allen organischen Lösungsmitteln aus. Es schmilzt unter Zersetzung bei $108^{\circ}$. Zum Unterschied von der gleich zu erwähnenden farblosen Modifikation will ich die blaue $\alpha$-Nitrosochlorid nennen. Nach kurzer Zeit nämlich, oft schon nach wenigen Stunden, geht die $a$-Modifikation des Santennitrosochlorids in eine weisse Modifikation über, die ich als $\beta$-Santennitrosochlorid bezeichnen will. Diese Polymerisation - denn mit einer solchen hat man es zweifellos zu thun - kündigt sich zunächst dadurch an, dass die schöne Farbe des $\alpha$-Nitrosochlorids antängt unscheinbarer zu werden, worauf dann die Umwandlung im Laufe weniger Tage vollendet ist. Die $\beta$-Modifikation zeichnet sich durch eine auffallende Unlöslichkeit in organischen Lösungsmitteln sowie durch eine erheblich grössere Beständigkeit aus. Beim erhitzen auf ca. $90^{\circ}$ färbt es sich intensiv blau unter Zurückverwandlung in die blaue $\alpha$-Modifikation, um sodann wie diese unter Zersetzung bei $108^{\circ}$ zu schmelzen.

Ueber sehr ähnliche Verhältnisse hat kürzlich W al lach') beim Pinol berichtet. Hier bildet sich zunächst ein farbloses, bimolekulares Nitrosochlorid, das bei steigender Temperatur in die monomolekulare, intensiv blaugefärbte Form übergeht. Beim Santen erhält man primär das blaue Nitrosochlorid, das sich schnell zu der farblosen Modifikation polymerisiert. Jedoch tritt auch hier beim Erwärmen wieder Spaltung in die blane Form ein.

Analyse des $\alpha$-Nitrosochlorids:

$0,2546 \mathrm{~g}$ gaben im Bombenrohr 0,1922 $\mathrm{g} \mathrm{Ag} \mathrm{Cl}$.

Gefunden: $\mathrm{Cl}=18,64 \%$. Ber. für $\mathrm{C}_{9} \mathrm{H}_{14} \mathrm{NOCl}: \mathrm{Cl}=18,89 \%$.

Santenuitrolamine.

Charakteristische, krystallisierte Nitrolamine konnten bei der Umsetzung des $\alpha$-Nitrosochlorids mit verschiedenen Basen nicht erhalten werden. Mit Piperidin resultierte lediglich ein grünlich gefärbtes Oel, Toluidin zersetzte das Nitrosochlorid völlig; mit Anilin und $\beta$-Naphtylamin wurden zwar dunkelgefärbte bezw. ziegelrote feste Reaktionsprodukte erhalten, dieselben sind aber kaum zu reinigen und nicht geeignet, das Santen zu charakterisieren.

\section{Santennitrosit.}

Auch mit salpetriger Säure liefert das Santen ein intensiv blau getärbtes, festes Additionsprodukt. Ueberschichtet man eine Lösung von $5,5 \mathrm{~g}$ Natriumnitrit in $15 \mathrm{~g}$ Wasser mit einer solchen von $2,5 \mathrm{~g}$ Santen in ca. $20 \mathrm{~g}$ Aether und tropft dann langsam eine Lösung von

1) Wallach, Ann. 306, 278. 
$3,9 \mathrm{~g}$ konzentrierter Salzsäure (ca. 33\%) ein, so bläut sich die ätherische Schicht nach kurzer Zeit und nach beendigter Reaktion hinterbleibt beim Verdunsten des mit Wasser gewaschenen Aethers eine erhebliche Menge (ca. $4 \mathrm{~g}$ ) eines blauen krystallinischen Körpers. Sein Verhalten gegenüber manchen Lösungsmitteln, wie Petroläther, deutet darauf hin, das kein einheitliches Produkt, sondern ein Gemenge eines blauen mit einer geringeren Menge eines grünen Körpers entstanden ist, zu deren Trennung noch keine Versuche unternommen wurden. Auch dieser nitrositartige Körper schien sich - vielleicht nur in noch nicht reinem Zustande - nach einigen Wochen zu zersetzen.

\section{Santenchlorhydrat.}

Sättigt man eine Lösung des Santens in etwa dem gleichen Gewicht trockenen Aethers vorsichtig mit trockner, gasförmiger Salzsäure, so hinterbleibt bei schnellem Verdunsten des Aethers ein festes, farbloses Hydrochlorid. Man lässt am besten den Aether schnell völl ig verdunsten, da das Chlorhydrat schon in Aetherdämpfen sofort zerfliesst, und presst es kurze Zeit auf Thon ab. Der Körper ist in Geruch und Flüchtigkeit kaum von Pinenchlorhydrat zu unterscheiden, auch gegen etwas erhöhte Temperatur sowie Spuren von Feuchtigkeit ist er sehr empfindlich. Um ihn in guter Ausbeute zu erhalten, muss man frisch destilliertes Santen verwenden. Das Hydrochlorid scheint sich leicht $\mathrm{zu}$ isomerisieren, da es nach wenigen Tagen sowobl an der Luft wie auch im Exsiccator sich verfliissigte. Den Schmelzpunkt konnte ich zu etwa $80^{\circ}$ ermitteln, indessen dürfte derselbe vielleicht noch etwas höher liegen.

Ein schon einige Tage altes und daher nicht mehr ganz einwandfreies Präparat des Santenhydrochlorids gab folgende Analyse:

$$
\text { Subst. 0,1876: } \mathrm{Ag} \mathrm{Cl}=0,1630 \mathrm{~g} \text {. }
$$

Gefunden: $\mathrm{Cl}=21,45 \%$. Ber. für $\mathrm{C}_{9} \mathrm{H}_{14} \cdot \mathrm{HCl}: \mathrm{Cl}=22,35 \%$.

Immerhin ist ersichtlich, dass Santen ein Monochlorhydrat bildet.

\section{Santentribromid.}

Zur Charakterisierung des Santens gut geeignet ist das Tribromid. Man erhält es, wenn man in eine trockene Chloroformlösung des Kohlenwasserstoffs, die durch eine gute Kältemischung gekühlt wird, die 2 Mol. entsprechende Menge Brom vorsichtig eintropft. Nach einiger Zeit beginnt eine allmählich stärker werdende Abspaltung von Bromwasserstoff. Ist alles Brom eingetragen, so lässt man den geringen Ueberschuss desselben zweckmässig zusammen mit dem Chloroform abdunsten. Es hinterbleibt ein schweres Oel, dass an einem kïhlen 
Ort nach wenigen Tagen tast völlig erstarrt. Die nach Abpressen auf kaltem Thon erhaltenen etwas klebrigen Krystalle schmelzen nach vorsichtigem Umkrystallisieren aus Methylalkohol bei $62-63^{\circ}$ und haben die Zusammensetzung $\mathrm{C}_{9} \mathrm{H}_{18} \mathrm{Br}_{3}$.

Analysen:

1) Subst. 0,1935: $\mathrm{H}_{2} \mathrm{O}=0,0671, \mathrm{CO}_{2}=0,2088 \mathrm{~g}$

2) $" 0,2064: \mathrm{AgBr}=0,3174 \mathrm{~g}$

3) $, 0,1906: \mathrm{AgBr}=0,2952$, Gefunden: $\mathrm{C}=29,43 \%, \quad \mathrm{H}=3,85 \%, \quad \mathrm{Br}=65,44 \%$

Ber. für $\mathrm{C}_{9} \mathrm{H}_{1 \mathrm{~B}} \mathrm{Br} \mathrm{r}_{3}: \mathrm{C}=29,92 \%, \mathrm{H}=3,60 \%, \mathrm{Br}=66,48$,

Oxydation des Santens.

Um eventl. einen Fingerzeig bezüglich der Konstitution des Santens zu bekommen, wurde eine geringe Menge desselberı oxydiert. Verbraucht wurde die etwa 5 Atomen Sauerstoff entsprechende Menge Permanganat. Aus der Oxydationslange konnte indessen nur eine sehr geringe Menge einer flüssigen, ziemlich wasserlöslichen Säure gewonnen werden, von deren Untersuchung wegen zu geringer Menge Abstand genommen werden musste.

\section{Ketone aus Sandelholzvorläufen.}

Die oben (Seite 367$)$ erhaltenen bei ca. $80-100^{\circ}(15 \mathrm{~mm})$ siedenden Fraktionen 8 und 9, die sich durch ein auffallend hohes spez. Gewicht auszeichneten, enthalten als Hauptbestandteil ein neues Keton $\mathrm{C}_{11} \mathrm{H}_{18} \mathrm{O}$. Zur Isolierung desselben empfiehlt sich sein Semikarbazon. Das Oxim war nicht nur beim Behandeln der Fraktionen mit überschüssigem Hydroxylamin schwer fest zu erhalten, sondern bildete auch bei der Abspaltung des Stickstoffs kein Keton zurïck. Mit alkoholischem Semikarbazid hingegen gab das rohe Keton schon nach kurzer Zeit ein krystallinisches Semikarbazon, das mit Petroläther gewaschen bei ca. $215^{\circ}$ schmolz. Die Menge des auskrystallisierenden Semikarbazons ist nur gering. Fällt man indessen die von dem Semikarbazon abfiltrierten Laugen nach mebreren Tagen mit Wasser aus, so erhält man eine erheblich grössere Menge Semikarbazon, das aber erheblich tiefer schmilzt. Aus $64 \mathrm{~g}$ der zwischen 80 und $110^{\circ}$ ( $15 \mathrm{~mm}$ Druck) siedenden Fraktionen wurden $7,1 \mathrm{~g}$ des hochschmelzenden und $41,5 \mathrm{~g}$ des niedriger schmelzenden Semikarbazons gewonnen. Diesen in reinem Zustande bei $224^{\circ}$ bezw. $175^{\circ}$ schmelzenden Semikarbazonen liegen verschiedene Ketone zu Grunde. 
Das bei der wiederholten Behandlung der Ketonfraktionen mit Semikarbazid hinterbleibende Produkt besitzt einen äusserst unangenehmen und intensiven Geruch, der fast an den gewisser Stickstoff basen erinnert. Eine Untersuchung dieses Körpers steht noch aus.

$$
\text { Santalon } \mathrm{C}_{11} \mathrm{H}_{10} \mathrm{O} \text {. }
$$

Von dem eben erwähnten niedrigschmelzenden und leichter in Alkohol löslichen Semikarbazon wurden $38 \mathrm{~g}$ bis zur völligen Geruchlosigeit mit einem Gemenge von Petroläther und wenig Aether gewaschen. Aus den restierenden $19 \mathrm{~g}$ wurden durch Zersetzen mit heisser verd. Schwefelsäure $10 \mathrm{~g}$ eines farblosen Ketons gewonnen, das im Geruch an Kampher und Thujon erinnerte. Aus dem beim Waschen in Lösung gehenden Semikarbazon kann bei entsprechender Aufarbeitung gleichfalls noch ein sehr reines Santalon gewonnen werden. Der Siedepunkt des reinen Santalons liegt bei $214-215^{\circ}$, bezw. $88-89^{\circ}$ unter $15 \mathrm{~mm}$ Druck, das spez. Gewicht bei $15^{\circ}$ ist 0,9906 und die optische Drehung im $100 \mathrm{~mm}$ Rohr $-62^{\circ}$.

\section{Analysen:}

1) Subst. $0,1215: \quad \mathrm{H}_{\mathbf{2}} \mathrm{O}=0,1136, \mathrm{CO}_{2}=0,3570 \mathrm{~g}$

2) n $0,1198: \mathrm{H}_{2} \mathrm{O}=0,1093, \mathrm{CO}_{2}=0,3536$ n

Gefunden: 1) $\mathrm{C}=80,13 \%, \quad \mathrm{H}=10,39 \%$

2) $\mathrm{C}=80,50$, $\mathrm{H}=10,14$,

Berechnet für $\mathrm{C}_{11} \mathrm{H}_{10} \mathrm{O}: \mathrm{C}=80,49, \mathrm{H}=9,76_{\text {n }}$

Da somit ein neues Keton $\mathrm{C}_{11} \mathrm{H}_{18} \mathrm{O}$ vorliegt, will ich es vorläufig als Santalon bezeichnen. Dasselbe ist isomer, aber nicht identisch mit dem von A. Hesse aus Jasminblütenöl isolierten Jasmon ${ }^{1}$ ).

\section{Sautalonoxim.}

Während die Darstellung eines festen Oxims aus dem nur durch Fraktionieren gereinigten Keton Schwierigkeiten bietet, gelingt dieselbe sehr leicht, wenn man von reinem Keton ausgeht. Löst man das wie oben beschrieben durch Semikarbazid gereinigte Santalon mit einem nicht zu kleinen Ueberschuss von Hydroxylaminchlorhydrat in wenig heissem Methylalkohol, setzt die theoretische Menge Aetzkali in wenig Methylalkohol gelöst hinzu, und kocht ca. 2-3 Stunden, so krystallisiert das entstandene Oxim zu einem erheblichen Teile schon beim Erkalten aus, oder kann in quantitativer Ausbeute durch Eingiessen in Eiswasser sofort fest erhalten werden. Es schmilzt, wiederholt ans Methylalkohol umkrystallisiert, bei $74,5-75,5^{0}$.

1) Hesse, Ber. 32, 2617. 
Analyse:

Subst. 0,1280: $\mathrm{H}_{2} \mathrm{O}=0,1130, \quad \mathrm{CO}_{2}=0,3457 \mathrm{~g}$.

Gefunden: $\mathrm{C}=73,66 \%, \mathrm{H}=9,81 \%$

Ber. für $\mathrm{C}_{11} \mathrm{H}_{16} \mathrm{NOH}: \mathrm{C}=73,74$ n $\mathrm{H}=9,50$ n

Santalonoxim giebt, mit verdünnter Schwefelsäure gekocht, kein Santalon zurück, sondern einen noch nicht näher untersuchten Körper von angenehm nitrilartigem Geruch. Zur Reinigung des Santalons ist also das Oxim nicht geeignet.

\section{Hydrobromsantalon.}

Das Santalon bildet, mit einer kalten, konzentrierten Lösung von Bromwasserstoff in Eisessig behandelt, ein Hydrobromid. Kocht man das von tuberschüssigem Bromwasserstoff befreite Hydrobromid mit einem Molekül Natriummethylat, so bekommt man ein nur noch geringe Spuren Brom enthaltendes Oel von starkem Kamphergeruch, welches mit überschüssigem Hydroxylamin nach der beim Santalonoxim angewandten Methode ein bei ca. $100^{\circ}$ schmelzendes Oxim liefert, dem anscheinend ein dem Santalon isomeres Keton zu Grunde liegen dürfte.

Hochschmelzendes Semikarbazon.

Das oben erwähnte bei $224^{\circ}$ schmelzende, in Alkohol schwer lösliche Semikarbazon aus den Ketonfraktionen lieferte mit Schwefelsäure zerlegt, aus $6,5 \mathrm{~g}-2,2 \mathrm{~g}$ eines nach Kampher riechenden Ketons, das indessen im Gegensatz zum Santalon Neigung zum Festwerden zeigte. Eine nähere Untersuchung steht auch hier noch aus.

Was die Menge des im Sandelholzöl enthaltenen Santalons betrifft, so konnte ich bei Untersuchung der Vorläufe zweier Portionen von je 300 Kilo Sandelholzöl einen Gehalt von ca. 0,045 bezw. 0,07\% Santalon feststellen. Die Menge des Ketons mit hochschmelzendem Semikarbazon dürfte etwa den 6. Teil derjenigen des Santalons ausmachen.

\section{Saure Bestandteile des Sandelholzöles.}

$$
\text { Teresantalsäure } \mathrm{C}_{10} \mathrm{H}_{14} \mathrm{O}_{2} \text {. }
$$

Wie schon oben erwähnt, wurden die Sandelholzölvorläufe vor weiterer Verarbeitung mit verdünnter Natronlauge behandelt, um denselben beigemengte Säuren zu entfernen.

Zur Reinigung dieser Säuren wurde ihre alkalische Lösung mit Aether durchgeschüttelt, das überschüssige Natron durch Einleiten von Kohlensäure abgestumpft und nochmals verschiedentlich ausgeäthert, wodurch auch eine sehr geringe Menge phenolartig riechender Körper entfernt werden konnte. Aus der Lauge wurden 
dann durch Ausfällen $98 \mathrm{~g}$ einer festen Säure gewonnen, die durch wiederholtes Umkrystallisieren auf den konstanten und glatten Smp. $157^{\circ}$ gebracht werden konnte. Die Säure krystallisiert aus Alkohol bei langsamem Verdunsten in gut ausgebildeten Prismen, die an der Basis in der Regel verwachsen sind. Sie destilliert unter $11 \mathrm{~mm}$ Druck bei ca. $150^{\circ}$ ohne Zersetzung. Charakteristisch sind ihre schwerlöslichen, weissen Ag- und $\mathrm{Pb}$-Salze, ein leicht dissozierendes Ammoniaksalz und ein in Natronlauge schwerlösliches Natronsalz. Die Săure besitzt die Formel $\mathrm{C}_{10} \mathrm{H}_{14} \mathrm{O}_{2}$.

\section{Analysen:}

Freie Säure: 1) Subst. 0,1864: $\mathrm{H}_{2} \mathrm{O}=0,1410, \mathrm{CO}_{2}=0,4904 \mathrm{~g}$ 2) $" 0,1378: \mathrm{H}_{2} \mathrm{O}=0,1070, \mathrm{CO}_{2}=0,3655$ " Ag-Salz: Subst. 0,2180: $\mathrm{H}_{2} \mathrm{O}=0,0962, \quad \mathrm{CO}_{2}=0,3525, \quad \mathrm{Ag}=0,0858 \mathrm{~g}$ Freie Säure, gefunden: 1) $\mathrm{C}=\mathbf{7 1 , 7 5} \%, \mathrm{H}=8,41 \%$

2) $\mathrm{C}=72,34$, $\mathrm{H}=8,63$, Ber. für " $\mathrm{C}_{10} \mathrm{H}_{14} \mathrm{O}_{2}$ : $\mathrm{C}=72,29, \mathrm{H}=8,43$, Ag-Salz, gefunden: $\mathrm{C}=44,10 \%, \mathrm{H}=4,90 \%, \mathrm{Ag}=39,36 \%$ Ber. für $\mathrm{C}_{10} \mathrm{H}_{18} \mathrm{O}_{2} \mathrm{Ag}: \quad \mathrm{C}=43,96, \quad \mathrm{H}=4,76, \quad \mathrm{Ag}=39,56$,

Die soeben mitgeteilten Eigenschaften der Säure lassen keinen Zweifel, dass dieselbe mit der inzwischen von Guerbet ${ }^{1}$ ) im Sandelholzöl aufgefundenen Teresantalsäure identisch ist, weswegen ich im folgenden diesen Namen beibehalten will. ${ }^{2}$ )

\section{Hydrochlorteresantalsäure.}

Obgleich man die Teresantalsäure ihrer grossen Beständigkeit gegenüber Permanganat für eine gesättigte halten sollte, gelingt es doch, z. B. Halogenwasserstoff an ihr Molekül zu addieren. Leitet man in eine Lösung von Teresantalsäure in wenig Methylalkohol ${ }^{\text {) }}$ ) - zuletzt unter Eiskühlung - trockne Salzsäure ein, so fällt bald ein krystallinisches Produkt aus, das zunächst noch aus unveränderter Teresantalsäure besteht. Nach ca. 24 stündigem Stehen indessen besitzen die ausgefallenen Krystalle einen bedeutend erhöhten Schmelzpunkt und erweisen sich als chlorhaltig. Saugt man sie nach der genannten Zeit $a b$, und lässt an der Luft trocknen, so erhält man bei

1) Guerbet, Comptes rendus 1900, I, 417.

2) Die Ausführung vorliegender Untersuchung erfolgte unabhängig von Guerbet, dessen Abhandlung im Februar 1900 orschien. Der die Teresantalsäure betr. Teil dieser Untersuchung war bereits im September 1899 vollendet. ebenso.

$\left.{ }^{3}\right)$ In äthylalkoholischer oder ätherischer Lösung verläuft die Reaktion 
$185-186^{\circ}$ unter Gasentwicklung schmelzende Krystalle, deren Schmelzpunkt durch Umkrystallisieren aus wenig Methylalkohol aut $193^{\circ}$ gebracht werden kann. Das Filtrat lässt man am besten bis zur Trockne eindunsten, da das Salzsäureadditionsprodukt durch Wasser langsam zersetzt wird. Als Nebenprodukt entstehen anscheinend geringe Mengen eines gechlorten Esters. Hydrochlorteresantalsäure ist leicht löslich in den meisten organischen Lösungsmitteln, schwerer löslich in Petroläther.

Sie hat die Zusammensetzung $\mathrm{C}_{10} \mathrm{H}_{14} \mathrm{O}_{2} \cdot \mathrm{HCl}$, wie sich aus folgenden Analysen ergiebt:

1) Subst. 0,2084: $\mathrm{Ag} \mathrm{Cl}=0,1522 \mathrm{~g}$

2) $, 0,1410: \quad \mathrm{H}_{2} \mathrm{O}=0,0966, \quad \mathrm{CO}_{2}=0,3018 \mathrm{~g}$

Gefunden: $\quad \mathrm{Cl}=18,03 \%, \mathrm{H}=7,61 \%, \mathrm{C}=58,38 \%$

Ber. für $\mathrm{C}_{10} \mathrm{H}_{14} \mathrm{O}_{2} \cdot \mathrm{HCl}$ : $\mathrm{Cl}=17,49$ n $\mathrm{H}=7,41, \quad \mathrm{C}=59,29$,

Beim Titrieren mit Alkali zeigt die Hydrochlorteresantalsänre, die 2 Moleküle desselben verbrauchen sollte, ein eigentïmliches Verhalten, das dadurch kompliziert wird, dass sie nicht nur, statt Teresantalsäure zurückzubilden, ein isomeres Lacton liefert, sondern auch - analog der Entstehung des Styrols aus Hydrobromzimmtsäure - anscheinend unter gleichzeitiger Kohlensäureabspaltung in einen Kohlenwasserstoff $\mathrm{C}_{9} \mathrm{H}_{14}$ übergeht.

Hydrochlorteresantalsäure neutralisiert nämlich in der Kälte nur 1 Mol. Alkali und auch bei längerem Kochen nur einen Bruchteil des zweiten Moleküls.

\section{Titration:}

Subst. 1,1606 $\mathrm{g}$ : verbr. kalt 5,8 $\mathrm{ccm} \mathrm{n-KOH,} \mathrm{nach} \mathrm{längerem} \mathrm{Erhitzen}$ noch $1,7 \mathrm{ccm}$.

Berechnet für 1,1606 $\mathrm{g} \mathrm{C}_{10} \mathrm{H}_{14} \mathrm{O}_{2} \cdot \mathrm{HCl}: 2 \mathrm{Mol} . \mathrm{KOH}=11,47 \mathrm{~cm}$ n-Lösung.

Teresantalsäurelacton.

Das eben erwähnte auffällige Verhalten der Hydrochlorteresantalsäure gegen Alkali veranlasste zu folgendem Versuch.

$1 \mathrm{~g}$ der sehr fein verriebenen Hydrochlorteresantalsäure wurde in ca. $50 \mathrm{ccm}$ einer verdünnten Natronlange eingetragen und sofort durchgeschüttelt. Es fand momentan Lösung der Krystalle statt und nach wenigen Sekunden schied sich ein neuer, fester Körper von borneolartigem Geruch ab. Von diesem wurden $0,8 \mathrm{~g}$ durch Abfiltrieren, Waschen und Trocknen rein erhalten. In einigen Fïllen - unter noch nicht aufgeklärten Bedingungen - entsteht bei obigem Verfahren ein Produkt, das schwerer fest wird und besser durch Ausäthern aufgearbeitet wird. Es wird nach dem Verdunsten des Aethers nicht sofort fest, sondern erst nach kurzer Zeit, nachdem ein flüssiges, sehr 
leicht flüchtiges, kohlenwasserstoffartiges Nebenprodukt gleichfalis verdunstet ist. Auf diesen Körper soll weiter unten eingegangen werden.

Der neutrale, feste Körper von borneolartigem Geruch schmilzt bei $103^{\circ}$ und ist zweifellos als ein Lacton anzusprechen, da er dem Ausgangmaterial, der Teresantalsäure isomer ist. Geringe Spuren von Chlor, die dem Körper hartnäckig anhaften, konnten nicht entfernt werden, da er durch stärker chlorentziehende Mittel verändert wurde; dieselben waren aber so unbedeutend, dass ihr Einfluss auf die Analysenzahlen nicht in Betracht kam.

$$
\begin{array}{r}
\text { A nalyse: } \\
\text { Subst. 0,1324: } \mathrm{H}_{2} \mathrm{O}=0,1026, \quad \mathrm{CO}_{2}=0,3510 \mathrm{~g} \\
\text { Gefunden: } \mathrm{C}=72,30 \%, \mathrm{H}=8,61 \% \\
\text { Ber. für } \mathrm{C}_{10} \mathrm{H}_{14} \mathrm{O}_{2}: \quad \mathrm{C}=72,29, \quad \mathrm{H}=8,43 \%
\end{array}
$$

Oxybydroditeresantalsäure.

Aus den zur Entchlorung der Hydrochlorteresantalsäure benutzten Natronlaugen konnten in allen Fällen durch ansänern mit Schwefelsäure geringe Mengen einer neuen, festen Säure erhalten werden, die zunächst als die dem Teresantalsäurelacton zugehörige Oxysäure angesprochen wurde. Der Schmelzpunkt der rohen Säure lag bei 140-145 ${ }^{\circ}$. Die Analysen der gereinigten Substanz ergaben indessen keine auf $\mathrm{C}_{10} \mathrm{H}_{18} \mathrm{O}_{3}$ stimmenden Werte. Daher wurden einige Gramm des Lactons durch mehrstündiges Kochen mit verd. Natronlauge aufgespalten, die Lösung, um Verunreinigungen zu entfernen, ausgeäthert und dann die zu erwartende Oxysäure ausgefällt und entsprechend aufgearbeitet. Das so gewonnene Material von verschiedenen Darstellungen wurde teils nur durch Umkrystallisieren aus Methylalkohol - wobei sich ein Schmelzpunkt von $143-144^{\circ}$ ergab - teils durch Darstellung des Silbersalzes und Waschen desselben mit Alkohol und Aether, teils durch regenerieren der freien Säure aus dem reinen Silbersalze und nachfolgendes, nochmaliges Umkrystallisieren gereinigt. Wiederholt ausgeführte Analysen gaben folgende Werte:

I. Säure, Nebenprodukt bei der Lacton-Darstellung.

1) Subst. 0,1212: $\mathrm{H}_{2} \mathrm{O}=0,0928, \mathrm{CO}_{2}=0,3033 \mathrm{~g}$ Ag-Salz desselben Produktes:

2) Subst. 0,2420: $\mathrm{H}_{2} \mathrm{O}=0,1096, \mathrm{CO}_{2}=0,3772, \mathrm{Ag}=0,0924 \mathrm{~g}$

2a) " 0,2666: $\quad-\quad-\quad \mathrm{Ag}=0,1012$,

II. Säure, aus reinem Lacton dargestellt; Ag-Salz derselben:

3) Subst. 0,2392: $\mathrm{H}_{2} \mathrm{O}=0,1084, \mathrm{CO}_{2}=0,3718, \mathrm{Ag}=0,0910 \mathrm{~g}$

3a) " 0,2531: $\mathrm{H}_{2} \mathrm{O}=0,1164, \mathrm{CO}_{2}=0,3960, \mathrm{Ag}=0,0960$ n III. Säure aus Lacton, durch Silbersalz gereinigt.

4) Subst. 0,1914: $\mathrm{H}_{2} \mathrm{O}=0,1516, \mathrm{CO}_{2}=0,4782 \mathrm{~g}$.

5) (In Form des Ag-Salzes analysiert): Subst. 0,1242: $\mathrm{H}_{2} \mathrm{O}=0,0598, \mathrm{CO}_{2}=0,1958, \mathrm{Ag}=0,0476 \mathrm{~g}$. 


\section{Freie Säure:}

\section{Gefunden:}

1) $\mathrm{C}=68,25 \%, \mathrm{H}=8,5 \mathrm{~L} \%$

4) $\mathrm{C}=68,14, \mathrm{H}=8,80$,

II. Ag-Salz.

2) $\mathrm{C}=42,51 \%, \mathrm{H}=5,03 \%, \mathrm{Ag}=38,18 \%$

2a) - $\quad-\mathrm{Ag}=37,96$,

3) $\mathrm{C}=42,39, \mathrm{H}=5,04, \mathrm{Ag}=38,04$,

3a) $\mathrm{C}=42,67, \quad \mathrm{H}=5,11, \mathrm{Ag}=37,93$,

5) $\mathrm{C}=43,00, \mathrm{H}=5,35, \mathrm{Ag}=38,33$,

Ber. für $\mathrm{C}_{10} \mathrm{H}_{16} \mathrm{O}_{8}$ : $\quad \mathrm{C}=65,22, \quad \mathrm{H}=8,70$,

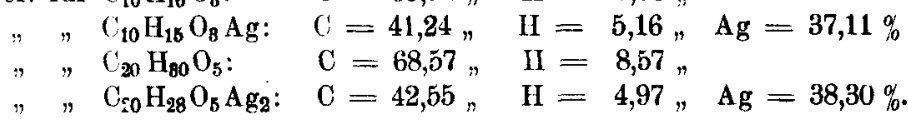

Diese gut übereinstimmenden Analysendaten kann man nur so auslegen, dass bei der Aufspaltung des Teresantalsäurelactons $\mathrm{C}_{10} \mathrm{H}_{14} \mathrm{O}_{2}$ mit Alkali nicht Oxyhydroteresantalsäure $\mathrm{C}_{10} \mathrm{H}_{10} \mathrm{O}_{3}$, sondern die 2-basische Oxyhydroditeresantalsäure $\left(\mathrm{C}_{10} \mathrm{H}_{14} \mathrm{O}_{2}\right)_{2} \cdot \mathrm{H}_{2} \mathrm{O}=\mathrm{C}_{18} \mathrm{H}_{28} \mathrm{O}\left(\mathrm{CO}_{2} \mathrm{H}\right)_{2}$ entsteht. Der Vorgang findet wohl so statt, dass 2 Moleküle der zunächst aus Hydrochlorteresantalsäure entstehenden Oxyhydroteresantalsäure unter Abspaltung eines Moleküls Wasser zu einem Molekül der 2-basischen Oxyhydroditeresantalsäure zusammentreten.

Einige mit geringen Mengen Teresantalsäure unternommene Versuche, durch Oxydation charakteristische Abbauprodukte zu erhalten, die einen Einblick in die Konstitution der Säure hätten gewähren können, hatten keinen Erfolg, da Permanganat die Teresantalsäure nur äusserst langsam angreift und Chromsäure nur flüssige Reaktionsprodukte lieferte. Daher wurde versucht, den der Săure zu Grunde liegenden Kohlenwasserstoff und das durch Destillation mit Calciumacetat zu erhaltende Keton darzustellen.

Kohlenwasserstoff $\mathrm{C}_{7} \mathrm{H}_{10}$.

$10 \mathrm{~g}$ Teresantalsäure und die etwas mehr als einem Molekül entsprechende Menge Essigsäure wurden zur Ketondarstellung zusammen in die Calciumsalze übergeführt und diese trocken destilliert. Das Destillat wog $6 \mathrm{~g}$, enthielt aber nur durch den Geruch wahrnehmbare Mengen Keton. Das mit Dampf übergetriebene, sehr flïchtige Reaktionsprodukt zeigte alle Eigenschaften eines Kohlenwasserstoffs, der sich auch durch Destillation des teresantalsauren Kalkes für sich allein bildete. Das Produkt siedete von $100-170^{\circ}$, die weitaus überwiegende Hauptfraktion lag zwischen 105 und $110^{\circ}$. Diese im Geruch an Lauch und Pinen zugleich erinnernde Fraktion wurde 2 mal über metallischem 
Natrium destilliert. Ihr spez. Gewicht bei $15^{\circ}$ war 0,818. Die Analyse gab nicht aut $\mathrm{C}_{9} \mathrm{H}_{14}$ - welche Formel schon nach dem Siedepunkt ausgeschlossen scheinen musste - sondern auf $\mathrm{C}_{7} \mathrm{H}_{10}$ stimmende Zahlen:

$$
\begin{aligned}
& \text { 1. Subst. 0,1222: } \mathrm{H}_{2} \mathrm{O}=0,1222, \mathrm{CO}_{2}=0,3988 \mathrm{~g} \\
& \text { 2. } 0,1238: \mathrm{H}_{2} \mathrm{O}=0,1250, \mathrm{CO}_{2}=0,4035, \\
& \text { Gefunden: 1. } \mathrm{C}=89,01 \%, \mathrm{H}=11,11 \% \\
& \text { 2. } \mathrm{C}=88,89, \mathrm{H}=11,22, \\
& \text { Ber. für } \mathrm{C}_{7} \mathrm{H}_{10}: \quad \mathrm{C}=89,36, \mathrm{H}=10,64,
\end{aligned}
$$

Der Kohlenwasserstoff $\mathrm{C}_{9} \mathrm{H}_{14}$, der primär entstanden sein müsste, war also anscheinend bei der Destillation in einen solchen $\mathrm{C}_{7} \mathrm{H}_{10}$ gespalten.

Abspaltung von Kohlensäure aus Teresantalsäure.

Die Darstellung des gesuchten Kohlenwasserstoffs $\mathrm{C}_{8} \mathrm{H}_{14}$ glückte auf einem anderen Wege. Schon bei der Abspaltung von Chlorwasserstoff aus Hydrochlorteresantalsäure war ein kohlenwasserstoffartiger Körper in geringer Menge beobachtet worden und auch bei einem Versuche, diese Säure durch Alkohol und konz. Schwefelsäure zu esterifizieren, war neben wenig Ester eine erhebliche Menge eines Nebenproduktes entstanden, in dem ein Kohlenwasserstoff vermutet wurde. Teresantalsäure wurde daher, in der Hoffnung, diesen Körper allein zu erhalten, der Einwirkung siedender Natronlange und siedender verdünnter Mineralsäuren ausgesetzt. Während siedende Natronlauge die Teresantalsäure nicht veränderte, verflüssigte letztere sich schon nach kurzem Kochen mit verd. Schwefelsäure und zwar konnte dabei das Entweichen eines Gases beobachtet werden, das sich als Kohlensäure erwies.

Es wurde daher eine etwas grössere Menge Teresantalsäure 3 Stunden lang unter Rückfluss mit dem gleichen Gewicht konz. Schwefelsäure - in der 8fachen Menge Wassers gelöst - gekocht und dann das Reaktionsprodukt mit Dampf abgeblasen. Aus $10 \mathrm{~g}$ Säure gingen dabei mit ca. $40 \mathrm{~g}$ Wasser $5,6 \mathrm{~g}$ eines leichten flüssigen Körpers über, der im Geruch von dem aus Sandelholzvorläufen isolierten Santen kaum zu unterscheiden war. Das Destillat wurde dann schwerer, und zuletzt ging ein krystallinischer nach Fenchylalkohol riechender Körper über. Letzterer bildete sich fast ausschliesslich, wenn die Teresantalsäure direkt aus verdünnter Schwefelsäure mit Dampf abgeblasen wurde; bei diesem Verfahren destillieren indessen geringe Mengen unveränderter Säure mit über. Aus $30 \mathrm{~g}$ Teresantalsäure wurden so $15 \mathrm{~g}$ des festen Körpers erhalten, der bei der Destillation folgende Fraktionen gab: 


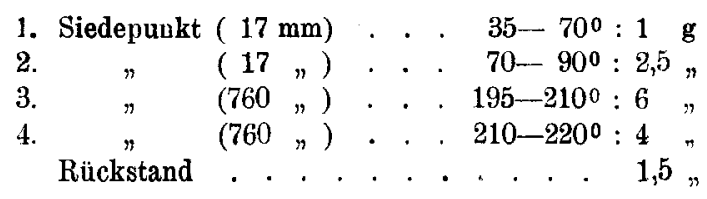

Fraktion 1 und 2 waren flüssig, die übrigen erstarrten sofort; der Schmelzpunkt der 4. Fraktion lag über $120^{\circ}$, wodurch eine Identität des Körpers mit Teresantalsäurelacton ausgeschlossen erscheint. Seine nähere Untersuchung steht noch aus.

\section{$\alpha-$ Santen.}

Um das eben erwähnte flïssige Reaktionsprodukt in etwas grösserer Menge darzustellen, wurden $20 \mathrm{~g}$ Teresantalsäure mit $160 \mathrm{~g}$ Wasser und $20 \mathrm{~g}$ Schwefelsäure 3 bis 4 Stunden unter Rückfluss gekocht und dann Dampf durchgeblasen. Das mit ca. $50 \mathrm{~g}$ Wasser übergehende Destillat, $11 \mathrm{~g}$, wurde für sich aufgefangen und die dann folgenden kleinen Fraktionen, die ein bedeutend höheres spez. Gewicht hatten, zurückgestellt. Von der Hauptfraktion wurden die mit Dampf am leichtesten flüchtigen Anteile, $8 \mathrm{~g}$, bei gew. Druck fraktioniert:

$\begin{array}{cl}\text { Siedepunkt }(760 \mathrm{~mm}): 140-1420: 2 \mathrm{~g} \\ " \\ " 142-145^{0}: 1,5 " \\ 145-1500: 1,5 " \\ " & 150-160^{\circ}: 0,5 " \\ " & 160-200^{\circ}: 2 "\end{array}$

Bei nochmaligem Fraktionieren siedeten $5 \mathrm{~g}$ bei $140-142^{\circ}$ (Hauptmenge bei $140^{\circ}$ ). Diese Fraktion, die den neuen Kohlenwasserstoff schon in sehr reiner Form darstellte, war im Geruch nicht von dem aus Vorläufen herausfraktionierten, oben beschriebenen Santen zu unterscheiden und auch seine sonstigen Eigenschaften und sein Verhalten sind dem des Santes so ähnlich, dass der Gedanke an eine Identität dieses Körpers - den ich vorläufig $\alpha$-Santen nennen will mit dem Santen nicht von der Hand zu weisen ist. Das $\alpha$-Santen ist inaktiv und besitzt ein spez. Gewicht von 0,870 bei $15^{\circ}$. Von dem a-Santen wurden zum Vergleich mit dem Santen einige feste Derivate dargestellt.

Tribrom- $\alpha$-Santen. 2,5 g $\alpha$-Santen wurden in der beim Santen beschriebenen Weise mit $2 \mathrm{Mol}$. Brom behandelt. Der Versuch verlief völlig analog der Bromierung des Santens. Das nach einigen Tagen fest erhaltene Bromid schmolz, einmal aus Methylalkohol umkrystallisiert, bei $59-61^{\circ}$, bei wiederholtem Umkrystallisieren fiel der Schmelzpunkt indessen auf $53-54^{\circ}$, während Tribromsanten bei $62-63^{\circ}$ schmilzt. 
Analyse:

Subst. 0,2168: $\mathrm{AgBr}=0,3314 \mathrm{~g}$.

$\begin{array}{cc}\text { Gefunden: } & \text { Berechnet für } \mathrm{C}_{8} \mathrm{H}_{18} \mathrm{Br}_{3}: \\ \mathrm{Br}=65,05 \% . & \mathrm{Br}=66,48 \% .\end{array}$

Hydrochlor- $\alpha$-Santen. 1,6 g $\alpha$-Santen wurden durch Lösen in ca. $2 \mathrm{~g}$ trockenem Aether und vorsichtiges Sättigen mit trockenem Salzsäuregas in das Hydrochlorid übergeführt. Auch dieses weist in Geruch, Flüchtigkeit etc. mit dem Santenhydrochlorid die grösste Aehnlichkeit auf; der Schmelzpunkt wurde allerdings etwas tiefer als beim Hydrochlorsanten, bei ca. $65^{\circ}$, gefunden. Berïcksichtigt man indessen, dass auch das Santenhydrochlorid sich ausserordentlich leicht verflüssigt bezw. seinen Schmelzpunkt erniedrigt und dass bei der geringen Quantität des $\alpha$-Santens eine völlige Reinigung nicht zu erzielen war, so wird man die z. T. ungenügenden Analysenresultate und die noch vorhandenen Unterschiede bei den Derivaten des Santens und $\alpha$-Santens nicht als erhebliche Gründe gegen die Identität dieser Körper geltend machen können. Analyse des Hydrochlorides:

Subst. $0,2944: \mathrm{AgCl}=0,2439 \mathrm{~g}$.

Gefunden:

$\mathrm{Cl}=20,45 \%$.
Berechnet für $\mathrm{C}_{9} \mathrm{H}_{14} \mathrm{HCl}$ :

$\mathrm{Cl}=22,35 \%$.

Ich stelle die Eigenschaften der beiden Kohlenwasserstoffe noch einmal vergleichend zusammen.

\begin{tabular}{|c|c|c|c|c|}
\hline & $\begin{array}{c}\text { Siedepunkt } \\
\text { (760 } \mathrm{mm})\end{array}$ & $\begin{array}{c}\text { Spez. Gew. } \\
\left(15^{\circ}\right)\end{array}$ & $\begin{array}{l}\text { Tribromid, } \\
\text { Schmp. }\end{array}$ & $\begin{array}{l}\text { Hydrochlorid, } \\
\text { Schmp. }\end{array}$ \\
\hline Santen . . . . . & $139-140^{\circ}$ & 0,871 & $62-63^{\circ}$ & $\left(\right.$ ca. $\left.80^{\circ}\right)$ \\
\hline$\alpha$-Santen . . & $140^{\circ}$ & 0,870 & $53-54^{0}$ & $\left(65^{0}\right)$ \\
\hline
\end{tabular}

Nimmt man die Identität des Santens mit dem $\alpha$-Santen an - wofür allerdings das Beweismaterial noch etwas dürftig ist -, so liegt die Annahme sehr nahe, dass das Santen im Sandelholz nicht fertig gebildet vorliegt, sondern erst bei der Destillation aus Teresantalsäure entsteht, da die Bedingungen für diese Reaktion bei der grossen Neigung der Teresantalsäure zur Kohlensäure-A bspaltung in der Regel vorhanden sein dürften.

Untersuchung von Verseifungslaugen des Sandelholzöles.

Die vorstehende Untersuchung der Teresantalsäure $\mathrm{C}_{10} \mathrm{H}_{14} \mathrm{O}_{2}$ wurde teils mit Material ausgeführt, das durch Ausschütteln der ersten 
Sandelholzölvorläufe mit verdünter Natronlauge direkt rein erhalten war, teils mit solchem, das durch Ausschütteln von Sandelholzöl mit Natriumbikarbonatlösung gewonnen war (vergl, unten).

Im Folgenden will ich kurz die Ergebnisse einer Untersuchung von Sandelholzöl-Verseifungslaugen beschreiben, die zu dem Zweck unternommen wurde, um die Anwesenheit anderer, die Teresantalsäure begleitender Säuren festzustellen. Die Teresantalsäure ist allergrösstenteils, wenn nicht ansschliesslich, in freiem Zustande im Sandelholzöl enthalten. Da sich in diesem indessen auch Ester, wenn auch in sehr geringer Menge, finden, so lag die Möglichkeit des Vorhandenseins einer oder mehrerer neuer Säuren vor.

80 Kilo rohes Sandelholzöl wurden zuerst sehr oft mit $10 \%$ iger Bikarbonatlösung, dann wiederholt mit verdünnter Natronlauge durchgeschüttelt und sodann mit heissem, alkoholischem Kali verseift. Die so gewonnenen alkalischen Laugen verdanke ich Herrn H. v. Soden, der die ebengenannten Operationen ausführen liess.

Die Aufarbeitung der Sodalaugen ergab $264 \mathrm{~g}$ Teresantalsäure, die nach einmaligem Umkrystallisieren rein war.

Aus den nach dem kalten Verfahren gewonnenen Natronlaugen wurden $233 \mathrm{~g}$ eines anfangs flüssigen Säuregemenges erhalten, das indessen nach einiger Zeit grösstenteils erstarrte. Sehr geringe Mengen intensiv riechender Phenole liessen sich durch Sättigen der Lauge mit Kohlensäure nicht rein abscheiden, da hierbei gleichfalls eine flüssige Säure mit in Freiheit gesetzt wurde. Die feste Säure, die etwa $\% / 3$ der Gesamtmenge ausmachte, erwies sich als Teresantalsäure, die sich also durch kohlensaure Alkalien nicht quantitativ ausschütteln lässt. Die flüssigen Anteile, die wohl zweifellos zum Teil aus der inzwischen von Guerbet') durch Dampfdestillation aus dem Säuregemenge isolierten Santalsäure $\mathrm{C}_{15} \cdot \mathrm{H}_{24} \mathrm{O}_{2}$ bestehen, sind wohl kaum einheitlicher Natur, da in ihnen anscheinend noch eine feste, wenn auch niedrigschmelzende und leicht $\mathrm{zu}$ verflüssigende fremde Säure enthalten ist.

Guerbet giebt seiner Santalsäure die Formel $\mathrm{C}_{15} \mathrm{H}_{24} \mathrm{O}_{2}$ und erwähnt ausdrücklich, dass sie aus ihren alkalischen Lösungen schon durch Kohlensäure ausgefällt werde. Wenn diese Formel zutrifft, so findet sich im Sandelholzöl zweifellos noch eine dritte Säure, die quantitativ erheblich sein muss. Ein schon längere Zeit vor Guerbets') Publikation aus den flüssigen, durch Kohlensäure ausfällbaren Anteilen des Säuregemenges bereitetes Silbersalz, in dem also santalsaures Silber vorgelegen haben müsste, wies nur einen Silbergehalt von $25,3 \%$ auf,

1) Guerbet, loc. cit. 
während $\mathrm{C}_{15} \mathrm{H}_{28} \mathrm{O}_{2} \mathrm{Ag} 31,49 \% \mathrm{Ag}$ erfordert. Dieses liesse also auf eine noch höher molekulare oder eine sauerstoffreichere Săure schliessen. Anderseits zeigte ein aus diesem Silbersalz dargestellter Aethylester bei $12 \mathrm{~mm}$ Druck einen fast glatten Siedepunkt von ca. $140-150^{\circ}$, eine Thatsache, die gegen ein sehr hohes Molekulargewicht der zu Grunde liegenden Säure spricht, und auch mit dem von $G$ uerbet für Santalsäure $\mathrm{C}_{15} \mathrm{H}_{24} \mathrm{O}_{2}$ angegebenen Siedepunkt von $212^{\circ}$ bei $20 \mathrm{~mm}$ Druck unvereinbar wäre.

Bei der heissen Verseifung der obengenannten 80 Kilo Sandelholzöl wurden $387 \mathrm{~g}$ auch nach längerer Zeit flüssigbleibende Säuren gewonnen, die auch hier noch geringe Mengen Phenole enthielten. Die nähere Untersuchung dieser Produkte steht noch aus.

Aus 80 Kilo Sandelholzöl wurden also im ganzen $884 \mathrm{~g}=1,15 \%$ Săuren erhalten, von denen $497 \mathrm{~g}=$ ca. $58,5 \%$ der Gesamtmenge in freiem Zustande vorhanden gewesen sein dürften. Die Menge der Teresantalsäure schätze ich auf ca. $419 \mathrm{~g}=47 \%$ der Gesamtmenge $=$ ca. $0,5 \%$ des Sandelholzöles, der Rest kommt auf Santalsäure und andere noch unbekannte Säuren, deren Natur noch aufzuklären ist.

Göttingen, 29. Mai 1900.

\title{
Schlussbemerkungen ther die quantitative Bestimmung des Santonins. ${ }^{1}$ )
}

\author{
Von Karl Thaeter.
}

(Eingegangen den 12. VI. 1900.)

In der ersten Erwiderung auf die meiner Bestimmungsmethode des Santonins in den Flores Cinae von Herrn Katz ${ }^{2}$ zu teil gewordene Beurteilung habe ich gezeigt, wie die derselben zugeschriebenen Fehler nur mangelhait begründet waren. Durch die neuerdings erfolgte Veröffentlichung von Herrn $\mathrm{Katz}^{8}$ ) werde ich veranlasst, den Nachweis zu liefern, dass zur Erreichung der von mir früher wiederholt angegebenen Resultate nur eine exakte, technische Ausführung der Methode gehört, welche - wie ein Vergleich der ersten mit den letzten nach

1) Die Frage der Santoninbestimmung in den Flores Cinae ist hiermit für diese Zeitschrift erledigt. Redaktion.

2) Dieses Archiv 237, Hett 4.

8) Ibidem 238, Heft 2. 